\section{CPS-135 TERIFLUNOMIDE AND DIMETHYLFUMARATE IN PATIENTS WITH RELAPSING-REMITTING MULTIPLE SCLEROSIS}

S Izquierdo Munoz*, AM López Gónzalez, ME Cárdaba García, L Enriquez Olivar, E Queipo García, MT Sánchez Sánchez. Hospital Clínico Universitario de Valladolid, Servicio de Farmacia, Valladolid, Spain

\subsection{6/ejhpharm-2019-eahpconf.284}

Background The local Pharmacy and Therapeutics Committee (PTC) approved Teriflunomide (TE) and Dimethylfumarate (DMF) for the treatment of relapsing-remitting multiple sclerosis (RRMS). These drugs were used in first-line treatment in adults with RRMS.

Purpose To evaluate the use of TE and DMF in RRMS patients, according to the protocol approved by the PTC and to calculate the treatment adherence to oral drugs against RRMS.

Material and methods A descriptive, observational and retrospective study was conducted from November 2013 until March 2018 in a General Teaching Hospital. Patients who received at least one dose of DMF and TE were included.

Collected data from medical records were: sex, age, Expanded Disability Status Scale (EDSS), previous treatments, therapeutic failure, adverse reactions and adherence to medicines. The treatment adherence was calculated by consulting the electronic dispensing register.

Results Fifty-six patients were included (75\% female, 25\% male), median age was 42 (26-61) years. 36/56 patients received TE, 20/56 DMF.

$40 / 55$ patients had received one or two previous treatments according to the protocol: interferon beta- $1 \mathrm{~b} 250 \mathrm{mcg}$ (20.2\%), interferon beta-1a $30 \mathrm{mcg}(36 \%)$, glatiramer acetate (20.2\%), interferon beta-1a $22 \mathrm{mcg}(8 \%)$ and interferon beta1a $44 \mathrm{mcg}(15.6 \%)$.

Fifteen patients began with oral treatment directly: 5/15 according to the protocol, 2/15 post-trial access, 3/15 needle phobia, 3/15 suspicion low adherence to parenteral treatments and $2 / 15$ others.

The average of previous treatments received per patient was $0.85 \pm 0.63$. Median time between start and end of the treatment with the parenteral immunomodulatory drug was 3 $(0-17.8)$ years. The average EDSS at the start of oral treatment was $2.08 \pm 0.87$. EDSS data were available in $57 / 76$ patients.

During the study period, $12 / 56$ patients discontinued treatment with oral immunomodulatory, 8/12 patients discontinued TE and 4/12 DMF. TE was discontinued in 6/12 patients for therapeutic failure and $2 / 12$ for adverse reaction, DMF for adverse reactions in $4 / 12$.

Treatment adherence to oral RRMS drugs was $99.9 \%$. Adherence of patients who discontinued treatment was $100 \%$. Conclusion Teriflunomide and Dimethylfumarate are drugs mainly prescribed for the treatment of RRMS patients who had previously received at least one parenteral immunomodulatory drug, in accordance with the local PTC and adherence was optimal with the new oral medicines.

\section{REFERENCES AND/OR ACKNOWLEDGEMENTS}

No conflict of interest.

\section{CPS-136 ANALYSIS OF THE USE OF OMALIZUMAB IN ORAL TOLERANCE INDUCTION FOR HIGH-RISK FOOD ALLERGIES IN CHILDREN}

${ }^{1} \mathrm{M}$ Larrosa García*, ${ }^{1} \mathrm{I}$ Jimenez-Lozano, ${ }^{2} \mathrm{C}$ Blasco-Valero, ${ }^{1} \mathrm{C}$ Canete-Ramirez, ${ }^{1} \mathrm{MT}$ GarrigaBaraut, ${ }^{1}$ BM Garcia-Palop, ${ }^{2}$ BR Vila-Indurain, ${ }^{1} \mathrm{~S}$ Clemente-Bautista, ${ }^{1} \mathrm{MJ}$ Cabanas-Poy. ${ }^{1}$ Vall d'Hebron University Hospital, Clinical Pharmacy, Barcelona, Spain; ${ }^{2}$ Vall d'Hebron University Hospital, Paediatric Allergology, Barcelona, Spain

\subsection{6/ejhpharm-2019-eahpconf.285}

Background Omalizumab-assisted oral-induced tolerance (oaOTI) has been proved to reduce the number of adverse events and shorten the time needed to reach tolerance compared to conventional OTI in patients with high-risk food allergy (hrFA). However, there are no established recommendations for omalizumab use for this indication.

Purpose The objective of this study was to describe the experience of oaOTI in patients with hrFA and its economic impact.

Material and methods This was an observational retrospective study including all paediatric patients with hrFA who underwent oaOTI in a tertiary care hospital. Patients initiated oaOTI in the case of previous OTI failure and/or anaphylaxis after allergen intake and/or specific $\mathrm{IgE}>100 \mathrm{UI} / \mathrm{mL}$. Demographic data, dosage, duration of treatment and clinical outcomes were obtained from the prescription and clinical data software.

Results Sixteen patients (50\% girls) with a median age of 13.5 (range 8-18) years' old were included: 12 were allergic to milk and four to eggs. Median basal IgE was 1142 (255-3,960) KUI/mL and $69 \%$ of patients had a specific IgE $>100 \mathrm{UI} / \mathrm{mL}$. Omalizumab was started at least 16 weeks before OTI initiation. Mean \pm standard deviation initial dose was $556 \pm 366 \mathrm{mg} / \mathrm{month}(75-1,200 \mathrm{mg})$. Dosage recommendations for omalizumab in allergic asthma included in the Summary of Product Characteristics were followed in eight patients: six patients received lower doses and one patient received higher doses than recommended (there were no recommendations for one patient with $\operatorname{IgE}>1,500 \mathrm{UI} / \mathrm{mL}$ ). All patients successfully completed OTI and omalizumab was then tapered to the minimum tolerated dose. Two patients were able to stop omalizumab after 44.7 and 61 months, 14 patients are still on omalizumab maintenance and the dose was reduced in $12(75 \%)$ patients. Anaphylaxis occurred in three patients during OTI. Omalizumab injection was well tolerated: only one case of headache and one case of rash were reported. Median monthly cost was $€ 1100$ (€ 738-€ 2,952)/patient, including the initial dose.

Conclusion oaOTI allowed patients with hrFA to acquire tolerance rapidly and safely, however, it had a great economic impact. Further research is needed to define how to reduce or interrupt omalizumab treatment in patients receiving the drug as an adjuvant to OTI.

\section{REFERENCE AND/OR ACKNOWLEDGEMENTS}

Labrosse $\mathrm{R}$, et al. The use of omalizumab in food oral immunotherapy. Archiv Immunolog Ther Experi 2016;65:189-99.

No conflict of interest. 